\title{
Linha de cuidados para gestantes com sífilis baseada na visão de enfermeiros
}

\author{
Care line for pregnant women with syphilis in the view of nurses
}

Michelle Andiara de Medeiros Araújo ${ }^{1}$, Giovanna Gabrielly Custódio Macêdo ${ }^{1}$, Gigliola Marcos Bernardo de Lima $^{1}$, Matheus Figueiredo Nogueira ${ }^{1}$, Débora Raquel Soares Guedes Trigueiro ${ }^{2}$, Janaína von Söhsten Trigueiro ${ }^{3}$

Objetivo: construir uma proposta de linha de cuidado para a gestante com sífilis a partir da visão de enfermeiros. Métodos: pesquisa qualitativa, realizada com sete enfermeiras da Atenção Primária à Saúde, por meio de um roteiro de entrevista semiestruturado com dados sociodemográficos, profissionais e questões norteadoras. Para análise dos dados utilizou-se a técnica de Análise de Conteúdo na modalidade temática. Resultados: foi construída uma unidade temática central e cinco categorias que abordam a atuação da Atenção Primária à Saúde, dificuldades e potencialidades da assistência, processo de enfermagem, interprofissionalidade e a idealização de um caminho de cuidados. Conclusão: resultados apontam a existência de um cenário de assistência à gestante com sífilis, contudo, com ações minimamente articuladas. Os enfermeiros apresentam dificuldades que podem interferir no cuidado prestado, como a frágil captação das gestantes e parceiros, e falta de envolvimento da gestão municipal.

Descritores: Enfermagem; Gestantes; Sífilis; Atenção Primária à Saúde.

Objective: to build a proposal of care line for pregnant women infected with syphilis from the perspective of nurses. Methods: this is a qualitative research conducted with seven Primary Health Care nurses, through semistructured interviews with aid of a script with sociodemographic and professional data and guiding questions. The technique of Content Analysis in the thematic modality was used for data analysis. Results: a central thematic unit and five categories were built, addressing the performance in Primary Health Care, difficulties and potentialities of care, the nursing process, interprofessionality, and the idealization of a care path. Conclusion: the results indicated the existence of a scenario of care to pregnant women infected with syphilis, but with minimally articulated actions. Nurses present difficulties that may interfere in the care provided, such as the fragile search for pregnant women and partners, and lack of involvement on the part of municipal management. Descriptors: Nursing; Pregnant Women; Syphilis; Primary Health Care.

\footnotetext{
${ }^{1}$ Universidade Federal de Campina Grande. Cuité, PB, Brasil.

${ }^{2}$ Faculdade de Enfermagem Nova Esperança. João Pessoa, PB, Brasil.

${ }^{3}$ Universidade Federal da Paraíba. João Pessoa, PB, Brasil.
} 


\section{Introdução}

As infecções maternas decorrentes de microorganismos que são passíveis de transmissão ao feto constituem uma das principais possibilidades para aumento e manutenção dos altos índices de morbimortalidade perinatal ${ }^{(1)}$. As maiores taxas de transmissão dessas infecções concentram-se na sífilis, uma doença sistêmica definida como problema de saúde pública em virtude da alta frequência de condução e graves desfechos para a gestação e o feto, como abortamentos, perdas fetais tardias e óbitos neonatais ${ }^{(2-3)}$.

Segundo o Boletim Epidemiológico de Sífilis 2018, estima-se que 49 mil parturientes tiveram o diagnóstico de sífilis, resultando em aproximadamente 24.666 nascidos vivos com sífilis congênita e 206 óbitos em virtude de sua ocorrência ${ }^{(3)}$. Em proporção mundial, seis milhões de novos casos de sífilis ocorrem globalmente, sendo em sua maioria localizados em países de baixa e média renda ${ }^{(4)}$.

Os impactos pessoais, familiares e sociais decorrentes da amplitude dessa doença exigem uma assistência multidimensional ao binômio mãe-feto que contemple todos os aspectos inerentes ao processo de cuidar. Nessa perspectiva, a possibilidade da construção de uma linha de cuidados abrange o trabalho em rede entre diferentes profissionais, em diversos serviços de saúde e setores sociais(5).

A Atenção Primária à Saúde assume o cenário ideal para que esse cuidado seja ampliado com base em atividades preventivas e não somente assistenciais e/ou administrativas ${ }^{(6)}$. 0 pré-natal, em consoante ao Programa de Humanização do Pré-Natal e Nascimento, apresenta-se na linha de frente do rastreamento, diagnóstico precoce e terapêutica hábil dos casos de sífilis na gestação. Estabelece as bases para a promoção de uma assistência de qualidade à gestante e ao feto/recém-nascido, além de proporcionar um conjunto de exames laboratoriais, imunobiológicos, classificações de risco e referências a outros níveis de atenção ${ }^{(7)}$.

Perante a ampla atuação do enfermeiro nesse nível de atenção, destacando sua participação na gestão do cuidado e na oferta da assistência à saúde da mulher e da criança em todas suas interfaces, como também a proporção dos danos decorrentes da sífilis e sua característica prevenível, o estudo se baseou nas seguintes questões norteadoras: Quais cuidados são prestados pelos enfermeiros da Atenção Primária à Saúde às gestantes com sífilis? Como está estruturada a Rede de Atenção à Saúde à gestante com sífilis no município?

A proposta de construção de uma linha de cuidados específica é lançada como método de qualificação e resolutividade da assistência à saúde, baseando-se em decorrência de a sífilis congênita compor o quadro de causas de morbimortalidade perinatal evitáveis, como também em virtude do cenário de fragilidade no aproveitamento das oportunidades de triagem, diagnóstico e tratamento, que corrobora para a prestação de cuidados pouco efetivos e sem qualidade.

Dessa maneira, objetivou-se construir uma proposta de linha de cuidado para a gestante com sífilis a partir da visão de enfermeiros atuantes na Atenção Primária à Saúde.

\section{Métodos}

Estudo qualitativo que englobou todas as nove Unidades Básicas de Saúde da Família do município de Cuité, Curimataú paraibano, com distribuição de cinco na zona urbana e quatro na zona rural, totalizando assim nove enfermeiras atuantes nas unidades investigadas.

Após levantamento do quantitativo de profissionais, considerou-se os seguintes critérios de inclusão: I) atuar em uma das Unidades Básicas de Saúde da Família do município; II) não estar afastado do exercício profissional durante o período de coletas da pesquisa. Houveram duas recusas, tornando a amostra total de sete enfermeiras.

As entrevistas foram realizadas durante os meses de novembro e dezembro de 2017, com agendamento em data, horário e local de preferência das 
participantes, com duração média de 5 a 20 minutos.

A coleta de dados foi mediada por um roteiro de entrevista semiestruturado criado pelos pesquisadores, que abordou questionamentos acerca de dados sociodemográficos, dados profissionais e questões norteadoras referentes à temática pesquisada. Os dados sociodemográficos incluíram sexo, idade e estado civil, enquanto os dados profissionais englobaram tempo de formação, tempo de atuação na Atenção Primária à Saúde, titulação e capacitação relacionada à sífilis. As questões norteadoras se basearam na percepção sobre a atual rede de atenção à gestante diagnosticada com sífilis no município, assistência profissional ofertada à gestante diagnosticada com sífilis, desenvolvimento do processo de enfermagem no acompanhamento da gestante diagnosticada com sífilis e dispositivos necessários para a construção de uma linha de cuidado à gestante diagnosticada com sífilis.

As informações obtidas durante a entrevista foram gravadas e, posteriormente, transcritas e analisadas. Para efetivação desta última etapa, empregou-se a técnica de Análise de Conteúdo, por meio da modalidade temática. A sistematização das entrevistas constituiu-se a partir da construção de uma unidade temática central e de categorias. Essa técnica metodológica adota as seguintes fases: pré-análise, exploração do material e inferência e interpretação dos resultados ${ }^{(8)}$.

Mediante análise minuciosa das falas das participantes da pesquisa, nomeou-se uma Unidade Temática Central: Conhecimento das enfermeiras da Atenção Primária à Saúde acerca da Sífilis na gestação. A partir dela, cinco categorias foram denominadas: A Atenção Básica como acesso primordial para efetivação da linha de cuidado da sífilis na gestação; As dificuldades/potencialidades do cuidado à gestante com sífilis; Necessidade de fortalecimento do processo de enfermagem para a sistematização da assistência às gestantes acometidas por sífilis; Interprofissionalidade e sua importância junto à gestante com sífilis e Um caminho de possibilidades para efetivação do cuidado. A linha de cuidados foi construída pelos pes- quisadores com base nos depoimentos das enfermeiras, sendo ressaltadas suas opiniões e necessidades, assim como as particularidades do serviço. Ademais, algumas ações e serviços de atenção foram implementados à construção, de modo a incluir iniciativas sociais e de proteção não mencionadas.

A pesquisa seguiu os princípios da Resolução 466/2012 do Conselho Nacional de Saúde, tendo sua coleta de dados precedida da submissão ao Comitê de Ética em Pesquisa do Hospital Universitário Alcides Carneiro, com parecer no 2.382 .256 e Certificado de Apresentação para Apreciação Ética no 76904417.3.0000.5182. Durante a interpretação dos resultados, atribuiu-se a cada participante o codinome de uma das joias preciosas a seguir: Diamante, Rubi, Safira, Esmeralda, Água Marinha, Ametista e Turmalina.

\section{Resultados}

A amostra foi composta em sua totalidade por participantes do gênero feminino; a idade variou de 23 a 42 anos, com média de 30 anos. Salienta-se que uma das participantes se negou a verbalizar a idade, sendo respeitada a sua decisão. No que diz respeito ao estado civil, cinco participantes se declararam casadas, enquanto apenas duas afirmaram possuir união não oficializada.

Em âmbito profissional, a maioria concluiu a graduação a menos de 10 anos e apenas duas profissionais apresentaram término superior a 10 anos. 0 período de atuação na Atenção Primária à Saúde variou de 11 meses a 17 anos. Em relação à titulação, uma participante apresentou pós-graduação Stricto Sensu (mestrado), enquanto quatro afirmaram possuir pós-graduação Lato Sensu (especialização) e uma em andamento. Apenas uma participante alegou ter capacitação direcionada à sífilis, especificamente a abordagens sindrômicas relacionadas à sintomatologia das infecções sexualmente transmissíveis.

0 restante afirmou ter realizado o curso para capacitação à realização de testes rápidos por meio do 
Telelab, o qual se configura como programa de educação permanente do Ministério da Saúde que disponibiliza cursos gratuitos virtualmente, tendo como público-alvo os profissionais da área da saúde.

\section{A Atenção Básica como acesso primordial para efetivação da linha de cuidado da sífilis na gesta- ção}

O Sistema de Atenção à Saúde busca garantir a integralidade do cuidado direcionado também ao binômio mãe/bebê e, para esse cuidado ser efetivado, os profissionais de saúde devem oferecê-lo de maneira contínua, de modo que o atendimento não seja fragmentado. A eliminação da sífilis depende da qualificação na assistência, principalmente da equipe inserida na Atenção Primária à Saúde, o qual se direciona como porta de entrada dessa Rede. Tal afirmação se enquadra na fala da entrevistada, a seguir: Bom... é... a porta de entrada né como atenção básica... o Programa Saúde da Família ele tem esse poder de ter o acesso e o acompanhamento a todas essas gestantes e aquelas que não têm o acompanhamento, que porventura elas, as faltosas, as que não querem fazer pré natal, se recusa... a gente consegue ter esse domínio (Diamante).

\section{As dificuldades/potencialidades do cuidado à ges- tante com sífilis}

A captação precoce das gestantes por meio dos Agentes Comunitários de Saúde e a utilização de redes sociais para manter a comunicação com as usuárias se configuram como potencialidades enfatizadas pelas enfermeiras. Em contrapartida, apesar da variedade de dispositivos que facilitam a adesão das gestantes às consultas, são apontadas algumas dificuldades que acabam interrompendo a continuidade do cuidado durante o pré-natal, como dificuldade na realização do teste rápido tanto pela gestante, quanto, sobretudo, pelos parceiros: Para facilitar que eu acho que é uma potencialidade eu criei um grupo no WhatsApp com todas as gestantes da unidade... (Água Marinha). Eu consigo meus agentes de saúde detectar essas, todas as gestantes que eu tenho no meu território, e assim, eu consigo 100,0\% de acompanhamento delas (Esmeralda). O que a gente encontra de dificuldade ainda no diagnóstico da sífilis é.. mas em questão do parceiro não vir fazer o teste... a mulher geralmente ela participa mais... (Turmalina). É quando a paciente não comparece para o teste rápido agendado. Teve um caso de sífilis congênita, porque ela não compareceu ao teste rápido agendado... (Rubi).

\section{Necessidade de fortalecimento do processo de en- fermagem para a sistematização da assistência às gestantes acometidas por sífilis}

A partir do questionamento de como o processo de enfermagem são desenvolvidos no acompanhamento a gestantes com sífilis, temos as seguintes afirmações: Através das consultas, é... do pré-natal, mas também através do acompanhamento familiar... a gente tem que... é, investigar o parceiro é... deixe eu ver o que mais... garantir que ela faça o tratamento corretamente... acho que é isso! (Ametista).Eu não tenho como responder essa agora... o processo de enfermagem seria mais o, o acompanhamento né? (Esmeralda).

Conforme exposto nos discursos acima, o processo de enfermagem não é efetivamente implementado, o que pode fragilizar a assistência prestada à gestante com sífilis. Apenas uma enfermeira falou como funciona a sistematização da assistência de enfermagem no cuidado à gestante com sífilis: Fiz todos os acompanhamentos no próprio prontuário de enfermagem, com a solicitação dos exames, com o acompanhamento... a gente fez toda a sistematização da assistência de enfermagem... a partir daquele, né, da, da... sistematização que eu fiz ali, é que vai ficar também registrado, né... até para outros profissionais que chegarem, que foi que aconteceu com essa minha gestante, ela teve uma segunda gravidez $e$ já não era mais eu... era uma outra enfermeira (Diamante).

\section{Interprofissionalidade e sua importância junto à gestante com sífilis}

O Núcleo Ampliado de Saúde da Família se apresenta como uma das principais alternativas para a interprofissionalidade, conforme as afirmações a seguir: A gente já tem que ter um preparo daquela gestante, tem que pegar um suporte com o Núcleo Ampliado de Saúde da Família, 
um acompanhamento psicológico dessa gestante pra que ela consiga né trabalhar esse, esse problema (Turmalina). Também encaminha pra... pra outros serviços como, por exemplo, a psicóloga, pra que essa gestante se sinta amparada... é... precisa do apoio psicológico pelo medo que a doença traz mesmo com as informações que a gente dá (Ametista).

\section{Um caminho de possibilidades para efetivação do cuidado}

A partir do questionamento às enfermeiras acerca do que adicionariam a uma linha de cuidado com foco na atenção à gestante com sífilis do município de acordo com as necessidades da sua prática, destacam-se acompanhamento multidisciplinar, assistência à saúde mental, educação permanente dos profissionais e detecção precoce, prévia ao desejo gestacional nos discursos a seguir: 0 que poderia como linha de cuidado seria o acompanhamento com equipe multidisciplinar... Um projeto terapêutico singular pra essa gestante! (Rubi). Eu acho que tem que vim da secretaria de saúde, eu creio que tem que vim de lá... treinamento, cartilha... (Safira). Detecção precoce. Essa detecção precoce vai começar... é, nas mulheres que fazem planejamento familiar, é... fazendo os testes ainda... ainda durante os... quando elas manifestam o desejo de... de engravidar... (Ametista).

Com base nas necessidades, a linha de cuidados foi proposta da seguinte forma: por meio da busca ativa, realizada pelos agentes comunitários de saúde, as gestantes da área serão identificadas, para assim realizar um pré-natal de qualidade. Por meio dos exames de triagem, no momento em que o enfermeiro e o médico da Atenção Primária à Saúde registram um caso de sífilis, a gestante deverá ser encaminhada ao Centro de Referência de Alto Risco da região, localizado na cidade de Campina Grande, Paraíba.

Confirmado o diagnóstico, a gestante e o parceiro deverão ser acompanhados por uma equipe composta por enfermeiro, médico, farmacêutico, psicólogo e assistente social, integrando o Núcleo Ampliado de Saúde da Família e o Centro de Referência em Assistência Social, que em conjunto delinearão o Projeto Terapêutico Singular. Consoante a isso, há in- tersetorialidade e interprofissionalidade entre os dispositivos Núcleo Ampliado de Saúde da Família e Centro de Atenção Psicossocial na promoção e cuidado à saúde mental da gestante e família.

O enfermeiro norteará o cuidado à gestante por meio do processo de enfermagem, traçando diagnósticos e implementando intervenções, com a finalidade de minimizar riscos preveníveis, obter resultados e atender as necessidades da usuária.

O Centro de Atenção Psicossocial comporia a linha de cuidados caso a gestante apresentasse sinais de sofrimento psíquico, alterações em funções mentais e risco para suicídio.

\section{Discussão}

Como limitações da pesquisa cita-se o reduzido quantitativo de participantes, considerando o universo de um município com apenas nove Unidades Básicas de Saúde da Família.

A proposta de construção de uma linha de cuidados direcionada à gestante com sífilis se apresenta como possibilidade para efetivação de um cuidado integral e resolutivo que contemple todas as interfaces da usuária, como também auxilia a atuação dos serviços de atenção à saúde e assistência social ao nortear o processo de tomada de decisão.

Consoante aos achados deste estudo, pesquisas internacionais apontam que os principais desafios relacionados à eliminação da sífilis congênita são a necessidade de melhorar o cuidado pré-natal, aumentar a cobertura dos testes rápidos, treinar os profissionais de saúde acerca do diagnóstico, tratamento e acompanhamento, e ampliar o acesso aos testes de rastreio e aos medicamentos utilizados na linha de frente do tratamento $^{(9)}$.

Além disso, a inclusão de mecanismos que norteiam o desenvolvimento de boas práticas de assistência, das quais cita-se a formulação de linhas de cuidado, também apresenta entraves condicionados a gestão municipal, os usuários e o envolvimento dos profissionais. 
Nessa perspectiva, a reorganização das práticas nas unidades tem o intuito de fazer com que as redes de atenção acompanhem os usuários durante o fluxo de atendimento dentro do sistema com base na resolutividade ${ }^{(10)}$. Esse sistema de integração de Redes de Atenção à Saúde proporciona aos usuários um encaminhamento mútuo entre níveis de complexidade de serviços.

Os sistemas integrados fazem com que as referências/contrarreferências funcionem, tornando-se eficazes nos três níveis de atenção. A necessidade de referência/contrarreferência nos casos de sífilis na gestação é organizada por meio de uma Rede de Atenção à Saúde, recomendando-se a participação nos diferentes pontos de atenção, tendo-os como mecanismos importantes no processo do cuidado, o qual deve ser contínuo ${ }^{(11)}$.

0 presente estudo revela que existe sim um caminho no qual a gestante com sífilis percorre no município. Todas realizam os testes rápidos durante o acompanhamento pré-natal e as enfermeiras sabem como agir caso o exame seja reagente, de modo a tornar a Atenção Primária à Saúde um ponto imprescindível para a detecção e acompanhamento dos casos.

A resolutividade do acompanhamento pré-natal é mediada pela utilização de ferramentas pela equipe da saúde para a captação precoce das gestantes, de modo a garantir a realização do número mínimo de consultas, acesso a métodos diagnósticos e monitoramento do binômio mãe/bebê ${ }^{(12)}$. Porém, o principal entrave para o controle da sífilis congênita no Brasil é o não tratamento ou tratamento inadequado dos parceiros das gestantes. Autores afirmam que os serviços de saúde devem obter uma nova postura, a fim de favorecer o acolhimento e traçar estratégias junto ao casal, já que a reinfecção pode perpetuar a doença e o tratamento adequado é determinante para a quebra de cadeias das infecções sexualmente transmissíveis $^{(13)}$.

Observa-se ainda por meio dos discursos, que o processo de enfermagem não é efetivamente implementado junto as suas unidades, dificultando, assim, a assistência integral à gestante com sífilis. 0 enfermeiro possui um papel relevante quanto ao manejo adequado do cuidado às gestantes, nesse caso específico, a sistematização do cuidado é desenvolvida por meio da consulta do pré-natal.

A aplicabilidade da sistematização nos serviços de saúde é aquém da esperada. Como exemplos de falhas estão a falta de conhecimento dos profissionais em como realizar corretamente a sistematização e deficiência na formação acadêmica. Tal fato se configura como descompromisso do método assistencial, quando o desconhecimento gera desinteresse a não adesão do processo de enfermagem em sua rotina de trabalho ${ }^{(14-15)}$.

Apesar de não implementar o processo de enfermagem, o enfermeiro é consciente que, por meio dela, é planejado e organizado todo o cuidado. Embora a Teoria das Necessidades Humanas Básicas tenha sido implantada há muito tempo atrás e exigida dentro das Instituições de saúde brasileiras, percebe-se que ela ainda não é seguida à risca ${ }^{(14)}$. Dessa forma, os enfermeiros devem se comprometer em articular novas práticas e saberes baseados em princípios científicos, assim como reavaliar constantemente o seu processo de trabalho.

A intenção é que a Educação Permanente em Saúde seja o pilar para a efetivação da prática dos profissionais da Atenção Primária à Saúde no município, uma vez que resulta em melhorias na produção do trabalho e da assistência propriamente $\operatorname{dita}^{(16)}$. A Estratégia Saúde da Família deverá realizar também atividades para sensibilizar a comunidade acerca de como prevenir e lidar com a sífilis.

A Organização Pan-Americana da Saúde incentiva a introdução de treinamentos com o objetivo de melhorar o trabalho dos profissionais na Atenção Primária à Saúde ${ }^{(17)}$. É imperativo também que o profissional busque alternativas de aprimoramento apesar 
dos meios oferecidos pela gestão municipal.

A Farmácia Básica municipal entra na linha com a oferta de medicamentos utilizados no tratamento da sífilis, já que ela se destina exclusivamente a Atenção Primária à Saúde e a nível ambulatorial. Fornece ainda fármacos à criança acometida por sífilis congênita e com manifestações clínicas aparentes, tais como pneumonia, lesões de pele e problemas ósseos ${ }^{(17)}$.

Programas sociais como o Bolsa Família, Projovem Adolescente, Programa Criança Feliz e a Farmácia Popular poderão ser inclusos na linha a partir das necessidades da gestante e sua conjuntura familiar. Ademais, o Centro de Referência Especializado de Assistência Social interviria em caso de vulnerabilidades e risco de prejuízo à integridade física, mental e social da gestante. A inserção desses serviços objetiva a promoção de melhor qualidade de vida e seguridade social à gestante e consequentemente ao bebê, com base na proteção social como constituição do direito social universal $^{(18)}$.

Na perspectiva da seguridade de direitos sociais e de saúde, a implementação do Projeto Terapêutico Singular em todos os dispositivos de assistência efetiva a qualidade e resolutividade do cuidado, assim como ações intersetoriais e interprofissionais com abordagem integral e interdependente a partir da reorganização do processo de trabalho em diferentes níveis de atenção ${ }^{(19)}$.

\section{Conclusão}

Os resultados obtidos apontam a existência de um cenário de assistência à gestante com sífilis, contudo, com ações minimamente articuladas. Identificou-se que os enfermeiros deste estudo obtêm conhecimentos acerca da temática, no entanto, apresentam algumas dificuldades que tendem a interferir na assistência prestada, a exemplo da frágil captação e alcance das gestantes e parceiros, falta de educação continuada e permanente e até falta de envolvimento da gestão municipal.

\section{Colaborações}

Araújo MAM e Trigueiro JVS participaram da concepção, projeto, análise e interpretação dos dados, redação do artigo e revisão crítica relevante do conteúdo intelectual. Macêdo GGC, Lima GMB, Nogueira MF e Trigueiro DRSG contribuíram para a aprovação final da versão a ser publicada.

\section{Referências}

1. Pereira DAP, Maia BP, Seto IIC, Bichara CNC. Congenital infection in patients enrolled in mother child reference program. Rev Paraense Med [Internet]. 2015 [cited mar 20, 2019]; 29(1):31-5. Available from: http://files.bvs.br/ upload/S/0101-5907/2015/v29n1/a4655.pdf

2. Dantas LA, Jerônimo SHNM, Teixeira GA, Lopes TRG, Cassiano AN, Carvalho JBL. Perfil epidemiológico de sífilis adquirida diagnosticada e confirmada em hospital universitário materno infantil. Enfermería Glob. 2017; 16(2):227-36. doi: http://dx.doi.org/10.6018/eglobal.16.2.229371

3. Ministério da Saúde (BR). Secretaria de Vigilância em Saúde. Boletim Epidemiológico de Sífilis 2018. Brasília: Ministério da Saúde; 2018.

4. Trinh TT, Kamb ML, Luu M, Ham DC, Perez F. Syphilis testing practices in the Americas. Trop Med Int Health. 2017; 22(9):1196-203. doi: http://dx.doi.org/10.1111/tmi.12920

5. Gryschek ALFPL, Nichiata LYI, Fracolli LA, Oliveira MAF, Pinho PH. Building the women's health network towards constructing a care line for pregnant and puerperium women in the Regional Management Board of Alto Capivari - São Paulo. Rev Saúde Soc. 2014; 23(2):689-700. doi: dx.doi. org/10.1590/S0104-12902014000200027

6. Mororó RM, Lima VC, Frota MVV, Linhares MSC, Ribeiro SM, Martins MA. A percepção dos enfermeiros da Estratégia de Saúde da Família acerca do seguimento da sífilis congênita. Rev Saúde.com [Internet]. 2015 [citado 2019 abr 20]; 11(2):291-302. Disponível em: http://www.uesb. br/revista/rsc/v11/v11N3a06.pdf 
7. Leal MC, Theme-Filha MM, Moura EC, Cecatti JG, Santos LMP. Atenção ao pré-natal e parto em mulheres usuárias do sistema público de saúde residentes na Amazônia Legal e no Nordeste, Brasil 2010. Rev Bras Matern Infant. 2015; 15(1):91-4. doi: http://dx.doi.org/10.1590/ S1519-38292015000100008

8. Bardin L. Análise de conteúdo. São Paulo: Edições 70; 2011.

9. Silveira MF, Leon RGP, Becerra F, Serruya SJ. Evolution towards the elimination of congenital syphilis in Latin America and the Caribbean: a multicountry analysis. Rev Panam Salud Publica. 2019; 43:e31. doi: https://doi.org/10.26633/ RPSP.2019.31

10. Peiter CC, Santos JLG, Lanzoni GMM, Mello ALSF, Costa MFBNA, Andrade SR. Healthcare networks: trends of knowledge development in Brazil. Esc Anna Nery. 2019; 23(1):e20180214. doi: http:// dx.doi.org/10.1590/2177-9465-ean-2018-0214

11. Lazarini FM, Barbosa BA. Educational intervention in Primary Care for the prevention of congenital syphili. Rev Latino-Am Enfermagem. 2017; 25:e2845. doi: http://dx.doi.org/10.1590/15188345.1612 .2845

12. Lopes ACMU, Araújo MAL, Vasconcelos LDPG, Uchoa FSV, Rocha HP, Santos JR. Implementation of fast tests for syphilis and HIV in prenatal care in Fortaleza-Ceará. Rev Bras Enferm. 2016; 69(1):62-5. doi: http://dx.doi.org/10.1590/00347167.2016690108i
13. Suto CSS, Silva DL, Almeida ES, Costa LEL, Evangelista TJ. Prenatal assistance to pregnant women diagnosed with syphilis. Rev Enferm Atenção Saúde [Internet]. 2016 [cited Apr 20, 2019]; 5(2):18-33. Available from: http://seer. uftm.edu.br/revistaeletronica/index.php/enfer/ article/view/1544/pdf

14. Soares MI, Resck ZMR, Terra FS, Camelo SHH. Systematization of nursing care: challenges and features to nurses in the care management. Esc Anna Nery. 2015; 19(1):47-53. doi: http://dx.doi. org/10.5935/1414-8145.20150007

15. Marinelli NP, Silva ARA, Silva DNO. Sistematização da assistência de enfermagem - desafios para a implantação. Rev Enferm Contemp. 2015; 4(2):25462. doi: http://dx.doi.org/2317-3378rec.v4i2.523

16. Silva CEM, Friedrich DBC, Farah BF, Silva KL. Continuing education and its interfaces with ambulatory care sensitive conditions. Rev Rene. 2017; 18(6):794-802. doi: http://dx.doi. org/10.15253/2175-6783.2017000600013

17. Cooper JM, Sánchez PJ. Congenital syphilis. Semin Perinatol. 2018; 42(3):176-84. doi: http://dx.doi. org/10.1053/j.semperi.2018.02.005

18. Couto BR. Assistência social: direito social ou benesse?. Serv Soc Soc. 2015; 124(1):665-77. doi: http://dx.doi.org/10.1590/0101-6628.045

19. Rocha EN, Lucena AF. Single Therapeutic Project and Nursing Process from an interdisciplinary care perspective. Rev Gaúcha Enferm. 2018; 39:e2017-0057. doi: dx.doi.org/10.1590/19831447.2018.2017-0057 